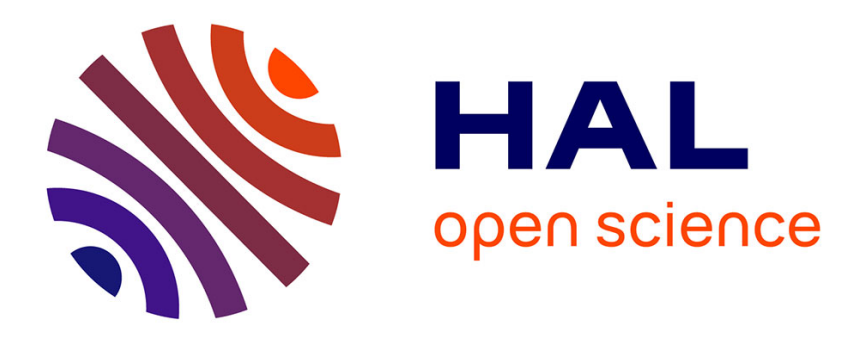

\title{
Error Probability Derivation in a Phonon-based Quantum Channel
}

\author{
Valeria Loscrì, Anna Maria Vegni
}

\section{To cite this version:}

Valeria Loscrì, Anna Maria Vegni. Error Probability Derivation in a Phonon-based Quantum Channel. QCIT'18 - Workshop on Quantum Communications and Information Technology, Dec 2018, Abu Dhabi, France. hal-01877153

\section{HAL Id: hal-01877153 https://hal.inria.fr/hal-01877153}

Submitted on 19 Sep 2018

HAL is a multi-disciplinary open access archive for the deposit and dissemination of scientific research documents, whether they are published or not. The documents may come from teaching and research institutions in France or abroad, or from public or private research centers.
L'archive ouverte pluridisciplinaire HAL, est destinée au dépôt et à la diffusion de documents scientifiques de niveau recherche, publiés ou non, émanant des établissements d'enseignement et de recherche français ou étrangers, des laboratoires publics ou privés. 


\section{Error Probability Derivation in a Phonon-based Quantum Channel}

\author{
Valeria Loscrí \\ Inria Lille - Nord Europe \\ Villeneuve d'Ascq, France \\ valeria.loscri@inria.fr
}

\author{
Anna Maria Vegni \\ Roma Tre University \\ Rome, Italy \\ annamaria.vegni@uniroma3.it
}

\begin{abstract}
Quantum communications are gaining more and more interest in the research community thanks to the recent advancements in nanotechnology. Indeed, quantum phenomena represent a natural direction for developing nanotechnology. The exploitation of quantum nature of information offers new potential solutions in the field of computing and networking, and extends the communication potentiality to levels that cannot be imagined in classical communication systems. Quantum communications can be realized in different ways. In this paper, we focus on the exploitation of quantum particles and quantum channels, in order to realize a data transmission system by means of phonons. First, we introduce the channel model of a phonon-based quantum system, and then derive the analysis of the error probability associated to such quantum channel. The application scenario is a biological environment, where phonons are exploited as information carriers. We have dealt a numerical evaluation in order to assess the performance of the quantum communication system. In particular, we have derived numerical results in terms of the error probability and the activity time, which represent how effective are phonons for communication purpose. We observe the frequency dependence of both error probability and activity time, thus allowing to tune the frequency for performance optimization.
\end{abstract}

Index Terms-Quantum channel, Phonons, Error probability

\section{INTRODUCTION}

Quantum communication theory exploits the quantum nature of information, allowing to envisage new potentialities for communication networks. Through quantum channels besides the classical information, quantum particles can be exploited as information carriers. Quantum communications represent the exchange of information encoded in quantum states of matter or quantum bits (known as qubits) between both nearby and distant quantum systems [1], [2]. Specific features rule the quantum communications, like the quantum entanglement, which occurs when the state of a particle interacts with another one and their quantum state cannot be described independently. It follows that quantum communication channels cannot be described by results based on classical information theory. Generally, the information is carried by quantum carriers and quantum states. A specific type of quantum particles are phonons that represent the quanta of mechanical vibrations or quanta of lattice vibration (i.e., acoustic excitations). Very recently, it has been observed the potentiality of phonons in the information processing field [3], [4]. Furthermore, due to pressure of Moore's law and Landauer entropy related to the limit of silicon-based electronic computing, great attention is being devoted to alternative methods of computation and communication. The implementation of a new communication paradigm based on the exploitation of phonons will pave the way to revolutionary applications in the context of communication technologies.

In [5], we considered phonons as quantum information carriers and quantum channels applied in a biological context. Phonons were generated through the interaction photonphonon, expressed by a Raman-Stokes scattering process; when a photon impacts on a sample of tissue, it generates a phonon. In [5] we also proved that the specific phononbased quantum communication system was similar to an optical quantum communication system. By applying quantum information theory results, we derived the capacity of the system, by assuming that quantum particles are used for classical information transmission.

Following the results in [5], in this paper we associate a basic communication scheme for characterizing the information transmission on a phonon-based quantum channel. Specifically, we consider an On Off Keying (OOK) scheme, where the generation of a phonon is associated to a qubit $\mid 1>$, while the absence of source excitation is associated to a qubit $\mid 0>$. Phonons are characterized by means of the relaxation time, meaning the time required for an excited phonon to return to its ground level energy that represents an equilibrium state for these particles. However, when the relaxation time is accounted in the quantum communication system, it represents a source of error. Indeed, an excited phonon represents the willingness to transmit this particle as a qubit $\mid 1>$. If the relaxation time is too small, it can be likely that the phonon will not be transmitted and this is considered as an error for the system that will consider a qubit $\mid 0>$ instead of $\mid 1>$. The phonons relaxation time depends both on the temperature and the frequency.

In the context of a biological environment (i.e., human tissue), we will consider a constant temperature, so the error probability derivation will only be based on the frequency. Furthermore, we will observe that the relaxation time affects the transmission effectiveness of the phonon-based quantum channel. For this aim, we will define the activity factor that a phonon will experience, expressed as the ratio of the relaxation time over the transmission window. The activity factor repre- 
sents the time interval where the phonon can be effectively transmitted over the quantum channel. To summarize, the main contributions of this paper can be summarized as $(i)$ the derivation of an analytical formulation of the error probability in dependence with the frequency, and (ii) the activity factor.

The rest of the paper is organized as follows. In Section II we formally describe the phonon-based quantum channel with the associated OOK scheme. Then, in Section III we derive the analytical formulation of the error probability associated to the phonon relaxation time. Section IV provides numerical results for the phonon-based quantum communication system in terms of error probability and the activity factor. Finally, we draw the conclusions at the end of the paper.

\section{Quantum Channel Model}

In this section, we characterize the data transmission in a phonon-based quantum channel as a stream of qubits $\mid 0>$ and $1>$, modulated through an OOK scheme.

We model the communication channel as an amplitude damping channel:

$$
E_{0}=\left[\begin{array}{cc}
1 & 0 \\
0 & \sqrt{1-P}
\end{array}\right] E_{1}=\left[\begin{array}{cc}
0 & \sqrt{P} \\
0 & 0
\end{array}\right]
$$

where the parameter $P$ indicates the probability of decaying from state $\mid 1>$ to $\mid 0>$ (i.e. in our case the probability of losing a phonon).

The amplitude-damping channel has been considered since it is a schematic model of the decay of an excited state.

We assume a time-based synchronization scheme at the transmitter side, then the transmission of a qubit $\mid 1>$ corresponds to the generation and emission of a phonon. Also, we are considering a biological environment, where phonons and photons are the result of electromagnetic waves emitted by a source, such as a common mobile phone. If no source is applied in a time slot, a qubit $\mid 0>$ is emitted, corresponding to the case of no excitation. The probability to send a qubit $\mid 0>$ is assumed to be equal to 1 .

The probability $p$ that the qubit $\mid 1>$ is correctly received by the receiver corresponds to the probability of a phonon to be emitted by a transmitter and to be received by a receiver. The failure probability of transmitting a qubit $\mid 1>$ when the transmitter is excited is $(1-p)$.

In this work, we focus on phonon lifetime and, more specifically, on multi-phonon relaxation time and derive the impact of relaxation mode on the error probability. In general, acoustic phonon relaxation time decreases with the increasing frequency. In [6], [7], Klemens predicted the relation between relaxation time $\tau$ and the frequency $\omega$ as $\tau \sim \omega^{-2}$.

\section{ERror Probability DERIVATION}

In this section we describe a noise model associated to the channel model as described in Section II. The theoretical derivation is general and can be applied to all the phononsbased quantum systems. However, in this paper we refer to multi-phonon relaxation time as the main source of error, while neglecting other sources of noise, such as the backscattering and re-thermalization effect. In practice, we account for the impact that the frequency plays into the relaxation time, by decreasing the effective number of phonons able to propagate and then propagate information.

Let us consider an OOK scheme to be applied in the amplitude damping channel. It is well-known that the distribution of the output signal for an OOK system at the receiver is [8]:

$$
p_{1}(r)=\frac{r}{\sigma^{2}} \exp \left(-\frac{r^{2}+A^{2}}{2 \sigma^{2}}\right) I_{0}\left(\frac{A r}{\sigma^{2}}\right),
$$

and

$$
p_{0}(r)=\frac{r}{\sigma^{2}} \exp \left(-\frac{r^{2}}{2 \sigma^{2}}\right),
$$

where $p_{1}$ represents the probability distribution when a qubit $\mid 1>$ is transmitted, $\sigma$ is the variance of the noise, $r$ is the received signal with amplitude $A, I_{0}$ is the zero-order modified Bessel function of the first kind, and $p_{0}$ represents the probability distribution associated to the transmission of a qubit $\mid 0>$.

In order to derive the error probability of the channel modeled as in Eq. 1, that a qubit $\mid 0>$ is received, while a qubit $\mid 1>$ has been transmitted, we can compute:

$$
P_{e}^{|0>,| 1>}=1-Q\left(\sqrt{2 \frac{E_{b}}{N_{0}}}, \frac{b}{\sigma}\right),
$$

where $Q$ is a Marcum- $Q$ function, $E_{b}$ is the energy associated to a phonon, $N_{0}$ is the noise energy, and $b$ represents a decision threshold that in our case coincides with the amount of energy needed to fill in the metastable state gap, then allowing the light emission, i.e.,

$$
b=\sigma \sqrt{2+\frac{E_{b}}{2 N_{0}}} .
$$

On the other side, the probability that a qubit $\mid 0>$ is sent but is detected as a qubit $\mid 1>$ can be neglected, since a qubit equal to $\mid 0>$ corresponds to the absence of source power (i.e., no generation of phonons), and for the moment we neglect the fact that there could be generation of phonons in absence of a specific source power. Theoretically, from Eq. (4) we can derive the error probability as

$$
P_{e}=\frac{1}{2}\left[\exp \left(-\frac{1}{2}\left(2+\frac{E_{b}}{2 N_{0}}\right)\right)\right] .
$$

In the specific context we are considering, the Signal-toNoise ratio (SNR) is related to the number of phonons. Indeed, as demonstrated in [5], we focus our analysis on a phononbased quantum channel, assuming an electromagnetic radiation incident on a human tissue sample. The estimations of $E_{b}$ and $N_{0}$ have to be related to the photon-phonon interaction referred to a sample of elements identified as cells (in a human tissue sample), in harmonic modes of vibrations. More specifically, the energy associated to each element/cell is

$$
E_{n}=\left(N+\frac{1}{2}\right) h \omega,
$$


where $E_{n}$ is the energy associated to the $n$-th state, $\omega=$ $2 \pi f$ is the frequency of a normal mode at which an element vibrates, and $h$ is the Planck constant. The process regulating the phonon generation by the means of a photon absorbed by a cell is the Raman-Stokes scattering process [9] and the associated phonon will have a frequency $f$. This means that the $n$-th cell will absorb an energy equal to

$$
E_{n}-E_{0}=n_{p} h \omega
$$

where $n_{p}$ is the number of generated phonons. An upper bound for the energy associated to each element is represented by $k_{B} T$, with $K_{B}$ representing the Boltzmann constant and $T$ the temperature.

This upper bound means that the number of phonons depends both by the temperature and the frequency. We assume that the maximum number of phonons is generated and that the phonons are characterized with the same frequency. This upper bound derives from the consideration that a cell cannot absorb a photon to achieve an energy higher than $k_{B} T$ representing the thermal noise. In practice, we assume that the number of phonons generated is equal to

$$
n_{p}=\frac{k_{B} T}{h f}-\frac{1}{2} .
$$

So, the final error probability will be derived by the consideration that the Hamiltonian operator associated to the channel can be represented as a set of plane wave modes with an associated SNR equal to

$$
S N R=\frac{2 E_{b}}{3 \hbar \delta \omega},
$$

where $\delta$ is the Dirac function and $\hbar$ is the reduced Planck constant.

Since we are evaluating the error probability due to the relaxation time of the phonons, we need to explicit the relation between the energy, the relaxation time (i.e., $\tau$ ) and the transmission time (i.e., $T_{s}$ ). Notice that the relaxation time represents the phonon lifetime i.e., the time needed to a phonon to return to its ground level energy. The relaxation time depends on the frequency and the temperature. However, in this work, we are assuming a constant temperature, so we do not consider this dependence. Concerning the frequency, we assume that

$$
\tau \sim \omega^{-\alpha}
$$

where $\alpha$ is a parameter that can assume different values, such as $\alpha=[0.5,1,1.5,1.85]$ as discussed in [10], [11].

By taking into account the relaxation time as a first order approximation, we can multiply the phonon energy by $\tau / T_{s}$, where $T_{s}$ represents the phonon transmission time. The ratio $\tau / T_{s}$ is defined as activity factor that represents the time interval where the phonon can be effectively transmitted over the quantum channel. Then, the error probability in Eq. (6) becomes:

$$
P_{e}(\tau)=\frac{1}{2}\left[\exp \left(-\frac{1}{2}\left(2+\frac{E_{b}\left(\tau / T_{s}\right)}{2 N_{0}}\right)\right)\right] .
$$

By considering that

$$
\frac{\tau}{T_{s}}=\frac{\delta}{2} \omega^{(1-\alpha)},
$$

the expression of the error probability in Eq. (12) becomes

$$
\begin{aligned}
& P_{e}(\tau)= \\
& = \begin{cases}\frac{1}{2}\left[\exp \left(-\frac{1}{2}\left(2+\frac{E_{b} \tau}{2 T_{s} N_{0}}\right)\right)\right], & \text { for }\left(\frac{\tau}{T_{s}} \mid P_{e}(\tau) \leqslant 1\right) \\
1, & \text { otherwise }\end{cases}
\end{aligned}
$$

and, more specifically, the expression of error probability can be written as

$$
P_{e}(\tau)=\frac{1}{2}\left[\exp \left(-\frac{1}{2}\left(2+\left(\frac{E_{b} \delta}{4 N_{0} \pi} \omega^{(1-\alpha)}\right)\right)\right)\right] .
$$

This expression allows a characterization of the error probability related with the phonon relaxation time and the frequency.

\section{PERFormance Evaluation}

In this section, we evaluate the error probability by focusing on a specific scenario, namely a biological tissue sample with a temperature of $37^{\circ} \mathrm{C}$. The temperature is considered constant and the impact of small variations are neglected since are not impacting considerably on the phonon relaxation time. The frequency window where activity has been demonstrated for the specific scenario considered is in the range $\left[10^{3}, 10^{12}\right] \mathrm{Hz}[5]$.

Initially, in Fig. 1 (a) we show the error probability in an ideal case (i.e., $\alpha=0$ ), that coincides with the minimum of the $P_{e}$. In fact, if we derive the error probability of Eq. (15) in respect to the parameter $\alpha$, we obtain:

$\frac{\partial P_{e}(\tau)}{\partial \alpha}=-\frac{1}{2}\left[\exp \left(-\frac{1}{2}\left(2+\frac{E_{b} \delta}{4 N_{0} \pi}\right)\right)\right] \omega^{(1-\alpha)} \ln (1-\alpha)$,

that is approximately equal to zero for $\alpha=0$. In general, the error probability is higher for higher values of $\alpha$ and in correspondence to higher frequencies.

Dealing with the numerical evaluation of the quantum communication system, three different intervals for $\alpha$ are of interest to be studied, i.e., (i) $\alpha<1$, (ii) $\alpha=0$, and (iii) $\alpha>1$. In Fig. 1 (b), we have evaluated $P_{e}$ for $\alpha=[0.3,0.5,0.7]$. The obtained results are very low, in the order of $10^{-4}$, with an increase for $\alpha \rightarrow 1$.

In the case $\alpha=1$, there is no dependence of the error probability in respect to the activity parameter. In this case, $P_{e}$ is directly dependent on the SNR, without considering the activity factor, and increases with the frequency as depicted in Fig. 1 (c). However, the obtained values are still very low.

Finally, in the case $\alpha>1$, the relaxation time is prohibitive for the transmission time $T_{s}$ and the error becomes too high. Fig. 1 (d) shows the dependence of error probability on frequency and given values of $\alpha$ in accordance to [6], [7]. We observe that for a fixed frequency, the error probability is higher when $\alpha$ is higher; also, for a fixed $\alpha$ it increases for increasing frequencies. This behavior is coherent by considering that for a fixed temperature, the error probability is 


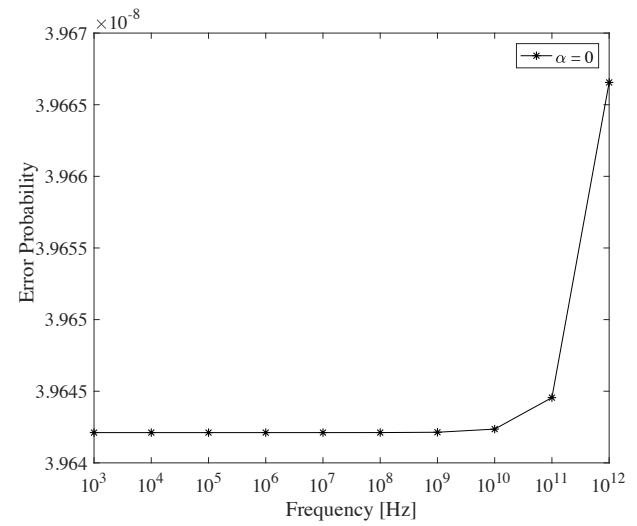

(a)

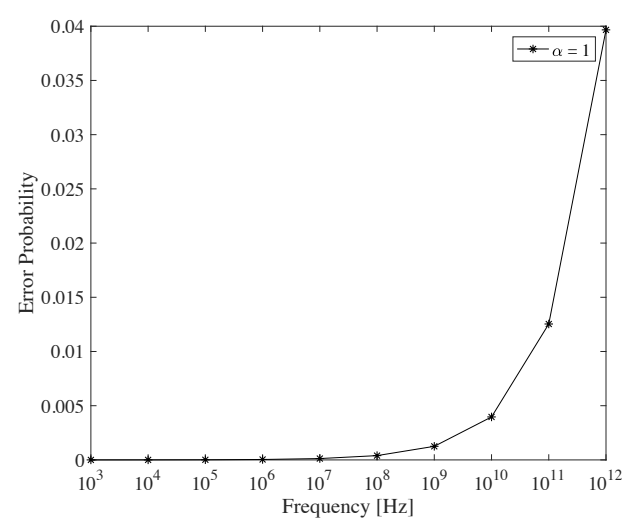

(c)

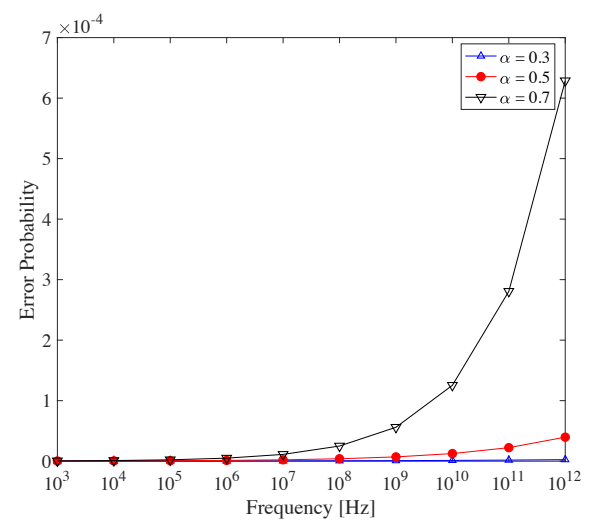

(b)

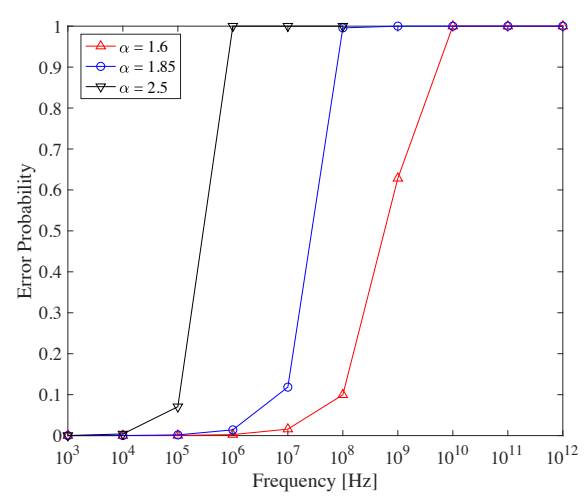

(d)

Fig. 1. Error probability of a quantum phonon-based channel versus frequency, for (a) $\alpha=0$, (b) $\alpha<1$, (c) $\alpha=1$, and (d) $\alpha>1$.

higher since there is a direct dependence of the transmission time with the frequency, since $T_{s}=2 \pi / \delta$. Moreover, the error probability associated to the phonon relaxation time increases as the factor $\alpha$ increases too. This is related to the fact that if $\tau$ is small, the time for transmitting a phonon is not enough and it results as a qubit $\mid 0>$ is received, even though a phonon has been emitted corresponding to the transmission of a qubit $\mid 1>$.

An interesting analysis to be dealt concerns the activity factor $\tau / T_{s}$, since it is associated to the phonon energy and consequently to the error probability. Indeed, we have a transmission time for the phonon that has to be considered in combination with the relaxation time. We observe that, the dependency by the frequency of $(i)$ the activity factor in terms of $\tau \sim \omega^{-\alpha}$ and (ii) the transmission time as $T_{s}=2 \pi /(\delta \omega)$, makes results not obvious.

In Fig. 2 we show the behavior of the activity factor with respect to the frequency $f$ and different values of $\alpha$. Again, the case $\alpha=0$ is an ideal condition where the relaxation time is really high in respect to the temporal evolution of the system. In this specific scenario, the activity factor is proportional to the frequency of phonons, and then we have very high values in the order of $10^{10}$ for higher values of frequency (see Fig. 2 (a)). In this case, as already shown in Fig. 1 (a), the error probability is increasing very slowly for higher frequencies (i.e., $P_{e}$ is almost constant); this is due to the fact that the transmission time is directly dependent on the frequency and then, the achieved qubit rate is higher in correspondence to higher frequencies, as observed in [5].

In Fig. 2 (b), for $\alpha=0.5$ the activity factor shows the same shape as for $\alpha=0$, but it is much smaller (i.e., in the order of $10^{4}$ ) than for $\alpha=0$. Coherently, the error associated to the communication system is higher for smaller values of the activity factor (see Fig. 1 (b)), since more phonons "vanish" before to be sent and this is translated as an error. Having high values of the activation factor means phonons have more time to live in order to be transmitted along the quantum channel.

On the other side, when $\alpha>1$, the shape of the curve changes and we have higher values of $\tau / T_{s}$ at lower frequencies, as depicted in Fig. 2 (c). By considering error probability evaluation for $\alpha>1$ (see Fig. 1 (d)), we notice that results are consistent with the values of the activity factor, since the error system converges rapidly to 1 . Indeed, the quantum particles are relaxing too fast to be able to carry the right information. Too fast is, of course, in respect to the feasibility of the information to be sent.

Finally, notice that the case $\alpha=1$ is a special case, since the factor $\tau / T_{s}$ does not depend anymore on the frequency 


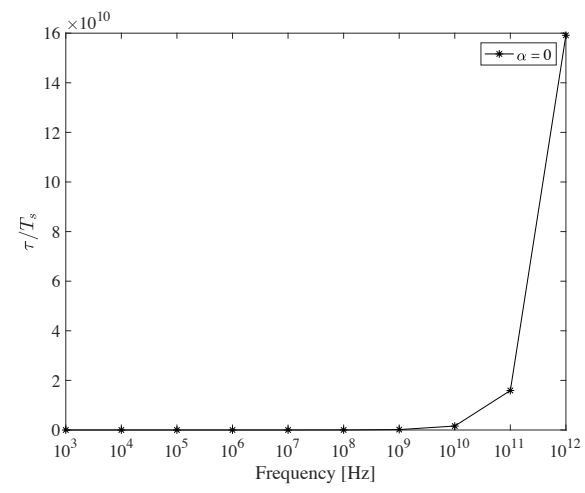

(a)

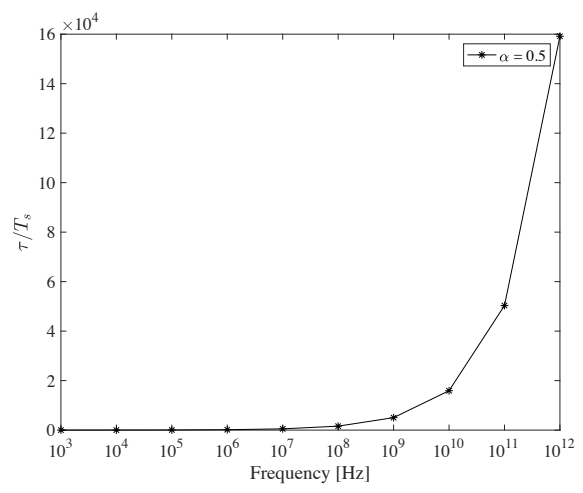

(b)

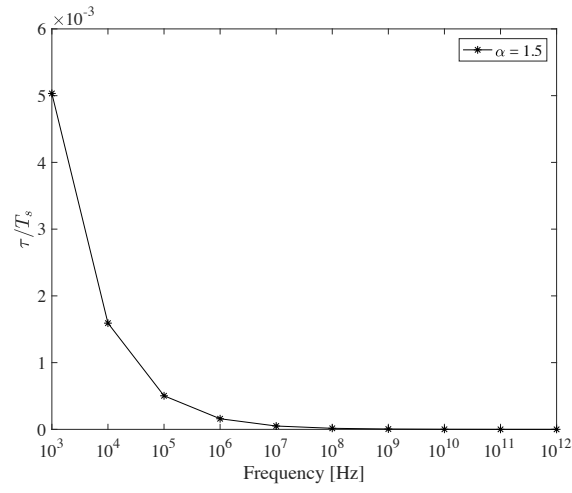

(c)

Fig. 2. Activity factor $\tau / T_{s}$ versus frequency, for (a) $\alpha=0$, (b) $\alpha=0.51$, and (c) $\alpha=1.5$, and (d) $\alpha>1$.

and it is constant. Nevertheless, the error probability depends by the frequency, since the SNR varies with the frequency.

\section{CONCLUSIONS}

Quantum communications are arousing more and more interest in the research community, since a great potentiality is associated to the exploitation of quantum particles and quantum states to carry out information data. The work dealt in this paper represents another step forward the phonon-based quantum networks. Specifically, we have considered quantum particles (i.e., phonons) and a quantum channel modeled as an amplitude-damping channel with error probability. We then derived the analytical expression of the error probability in a phonon-based quantum channel, by integrating the phonon relaxation time into the communication system. Achieved results have shown that at low frequencies the error probability is limited, even though the term accounting for the relaxation time and the transmission time has shown an evidence that the system behavior is complex and depends on several factors. Finally, simulation results have shown the behavior of the activity factor versus the frequency and different values of $\alpha$. High values of the activation time can guarantee a more effective quantum transmission by means of phonons.

\section{REFERENCES}

[1] M. Rahaman, "Multiplicative properties of quantum channels," Journal of Physics A: Mathematical and Theoretical, vol. 50, no. 34, 2017.
[2] L. Hanzo, H. Haas, S. Imre, D. O'Brien, M. Rupp, and L. Gyongyosi, "Wireless myths, realities, and futures: From $3 \mathrm{~g} / 4 \mathrm{~g}$ to optical and quantum wireless," Proceedings of the IEEE, vol. 100, no. Special Centennial Issue, pp. 1853-1888, May 2012.

[3] S. R. Sklan, "Splash, pop, sizzle: Information processing with phononic computing," AIP Advances, vol. 5, no. 5, p. 053302, 2015.

[4] L. Wang, G. Zhang, G. Wu, N. Yang, and B. Li, "Phononics: A new science and technology of controlling heat flow and processing information by phonons," in ASME, 14th International Heat Transfer Conference, vol. 8, 2010, pp. 601-619.

[5] V. Loscrí and A. M. Vegni, "Capacity evaluation of a quantum-based channel in a biological context," IEEE Transactions on NanoBioscience, vol. 15, no. 8, pp. 901-907, Dec 2016.

[6] P. Klemens, "The thermal conductivity of dielectric solids at low temperatures (theoretical)," Proceedings of the Royal Society of London A: Mathematical, Physical and Engineering Sciences, vol. 208, no. 1092, pp. 108-133, 1951. [Online]. Available: http://rspa.royalsocietypublishing.org/content/208/1092/108

[7] — Thermal Conductivity. Plenum Press: Springer, 1976.

[8] Q. Tang, S. K. S. Gupta, and L. Schwiebert, "Ber performance analysis of an on-off keying based minimum energy coding for energy constrained wireless sensor applications," in IEEE International Conference on Communications, 2005. ICC 2005. 2005, vol. 4, May 2005, pp. 27342738 Vol. 4.

[9] C. Raman and K. S. Krishnan, "The raman effect - inelastic insight," Nature, vol. 121, pp. 501-502, 1928.

[10] T. Feng, B. Qiu, and X. Ruan, "Anharmonicity and necessity of phonon eigenvectors in the phonon normal mode analysis," Journal of Applied Physics, vol. 117, no. 19, p. 195102, 2015.

[11] T. Feng and X. Ruan, "Prediction of spectral phonon mean free path and thermal conductivity with applications to thermoelectrics and thermal management: A review," Journal of Nanomaterials, vol. 2014, p. 25 pages, 2014. 